\title{
Global existence and nonexistence of solutions for quasilinear parabolic equation
}

Xianghui X ${ }^{1}$, Yong-Hoon Lee ${ }^{1}$ and Zhong Bo Fang ${ }^{2^{*}}$

${ }^{\text {*Correspondence: }}$ fangzb7777@hotmail.com

${ }^{2}$ School of Mathematical Sciences, Ocean University of China, Qingdao, 266100, P.R. China

Full list of author information is available at the end of the article

\begin{abstract}
This work is concerned with the global existence and nonexistence of solutions for a quasilinear parabolic equation with null Dirichlet boundary condition. Based on the Galerkin approximation technique and the theory of a family of potential wells, we obtain the invariant sets and vacuum isolating of global solutions including critical case, and we also give global nonexistence.
\end{abstract}

MSC: 35A01; 35B06; 35B08

Keywords: family of potential wells; global existence; nonexistence; vacuum isolating; critical value

\section{Introduction}

Our main interest lies in the following quasilinear $p$-Laplacian parabolic equation:

$$
\left|u_{t}\right|^{m-2} u_{t}=\operatorname{div}\left(|\nabla u|^{p-2} \nabla u\right)+b u^{1+q}, \quad x \in \Omega, t>0,
$$

subject to homogeneous Dirichlet boundary and initial conditions

$$
\begin{aligned}
& u(x, t)=0, \quad x \in \partial \Omega, t>0, \\
& u(x, 0)=u_{0}(x), \quad x \in \Omega,
\end{aligned}
$$

where $b>0, \Omega \subset R^{N}(N \geq 1)$ is a bounded domain with smooth boundary, $p<2+q<\infty$ if $N \leq p ; p<2+q<\frac{N p}{N-p}$ if $N>p$ and $2<m<2+q$. For simplicity, we denote $\|\cdot\|_{L^{p}(\Omega)}$ by $\|\cdot\|_{p}$ and $(u, v)=\int_{\Omega} u v d x$.

Many natural phenomena have been formulated as the nonlinear diffusive equation (1.1) such as the model of non-Newton flux in the mechanics of a fluid, the model of a population, biological species and filtration; we refer to $[1,2]$ and the references therein. In the non-Newtonian theory, the quantity $p$ is a characteristic of the medium. Media with $p>2$ are called dilatant fluids, while media with $p<2$ are called pseudoplastics. If $p=2$, they are Newtonian fluids.

There have been a lot of results on the global existence and nonexistence of solutions for nonlinear evolution equations and many effective methods have been developed such as the compactness method, the semi-group method, continuation of local solutions, the upper-lower solution method, and the concavity method; see [3-6]. Since the potential well was introduced by Sattinger [7] in order to prove the global existence of solutions for

\section{管 Springer}

@2014 Xu et al.; licensee Springer. This is an Open Access article distributed under the terms of the Creative Commons Attribution License (http://creativecommons.org/licenses/by/2.0), which permits unrestricted use, distribution, and reproduction in any medium, provided the original work is properly cited. 
nonlinear hyperbolic equations which do not necessarily have positive definite energy, it has become an important method to study the global existence and nonexistence of solutions for various nonlinear evolution equations. It is an effective method to get a positive definite energy through one term, controlled by the other term in the principal part of the equations [7-19]. For instance, Levine [8] investigated the initial boundary value problem of the linear heat conduction equation

$$
u_{t}=\Delta u, \quad x \in \Omega, t \in(0, T)
$$

with nonlinear boundary condition, and he obtained the global existence and nonexistence of weak solutions. Payne and Sattinger [9] investigated the initial boundary value problem of the semilinear hyperbolic equation with fully nonlinear term

$$
u_{t t}=\Delta u+f(u), \quad x \in \Omega, t>0
$$

and proved the weak solution blows up in finite time. They also extended the results to the initial boundary value problem of the corresponding parabolic equation. Tsutsumi [10] studied the homogeneous Dirichlet initial boundary value problem of the nonlinear parabolic equation

$$
u_{t}=\operatorname{div}\left(|\nabla u|^{p-2} \nabla u\right)+u^{1+q}, \quad x \in \Omega, t>0,
$$

and he obtained the sufficient conditions of the existence of global weak solutions and the solutions blow up in finite time for the case $p<2+q$. Later, Liu [11] proved the global existence of solutions of the homogeneous Dirichlet initial boundary value problem for (1.4) with critical initial conditions. Pang and Zhang [12] investigated the initial boundary value problem of the quasilinear parabolic equation

$$
\left|u_{t}\right|^{m-2} u_{t}=\Delta u+|u|^{q-1} u, \quad x \in \Omega, t>0
$$

and they obtained the sufficient conditions as regards global existence and nonexistence of solution by using a potential well method.

However, the potential wells used in these works were defined by the same method as Sattinger [7] and their results were similar. Until Liu [20] firstly introduced the theory of a family of potential wells, described the structure of potential wells and the estimates of the depth of potential wells. And he firstly found the phenomenon of vacuum isolating of solutions for nonlinear evolution equations. The study of applications about a family of potential wells has attracted more and more attention [20-25]. For instance, Liu and Zhao [21] not only proved the global existence and nonexistence of solutions, but they also obtained the vacuum isolating of solutions of the initial boundary value problem for semilinear hyperbolic equations and parabolic equations.

As far as we know, there are fewer papers on the global existence and nonexistence of weak solutions for nonlinear parabolic equations by using the theory of a family of potential wells. In particular, for our problem (1.1)-(1.3), the analysis of the structure and depth of the potential well, the invariant sets, the vacuum isolating of global solutions, and the question of the global existence of solutions with critical initial conditions are still open. 
It is difficult to obtain an a priori estimate of the approximate solution for the study of the existence of global solutions by using the general Galerkin approximation method, but the theory of the potential well often makes up for the defect. The combination of the two methods can be used to solve the existence of solutions effectively. Moreover, the study of the phenomenon of vacuum isolating will be helpful for us in studying the distribution of solutions in Sobolev space. But the depth of potential well $d$ for the problem (1.1)-(1.3) is usually very small etc. Our goal is to improve the theory of a family of potential wells for studying the global existence and nonexistence of solutions for our problem (1.1)-(1.3), including the critical case, and we further generalize the results in [10-12, 20].

The outline of the paper is as follows. In Section 2, we firstly give the definition of the weak solution for problem (1.1)-(1.3), and the definition and properties of a family of potential wells. Then we prove the global existence of solutions for problem (1.1)-(1.3) by using the Galerkin approximation technique and the theory of a family of potential wells in Section 3. The invariant sets of global solutions and vacuum isolating are obtained in Section 4. Then the sufficient condition of global nonexistence of solutions is given in Section 5. Finally, we give the result of global existence with critical initial conditions.

\section{Preliminaries}

Due to the degeneracy of (1.1), problem (1.1)-(1.3) has no classical solutions in general. We need to give the definition of the weak solution firstly.

Definition 1 A function $u=u(x, t)$ is called a weak solution of problem (1.1)-(1.3) on $\Omega \times$

$[0, T)$ if it satisfies the following conditions:

(1) $u \in L^{\infty}\left(0, T ; W_{0}^{1, p}(\Omega)\right), u_{t} \in L^{m}\left(0, T ; L^{m}(\Omega)\right)$;

(2) $\int_{0}^{t}\left(\left(\left|u_{t}\right|^{m-2} u_{t}, v\right)+\left(|\nabla u|^{p-2} \nabla u, \nabla v\right)\right) d \tau=\int_{0}^{t}\left(b u^{q+1}, v\right) d \tau$ for $\forall v \in W_{0}^{1, p}(\Omega), t \in[0, T)$;

(3) $u(x, 0)=u_{0}(x)$

where $T$ is either infinity or the limit of the existence interval of solution.

In order to study the problem (1.1)-(1.3), we also consider the auxiliary equation

$$
\left|u_{t}\right|^{m-2} u_{t}=\operatorname{div}\left(|\nabla u|^{p-2} \nabla u\right)+b \varphi(u), \quad x \in \Omega, t>0,
$$

where $\varphi(u)=\left\{u^{1+q}\right.$, if $u \geq 0$; 0 , if $\left.u<0\right\}$.

Next, we define the functionals $J(u), I(u)$, and the potential well $W$ as follows:

$$
\begin{aligned}
& J(u)=\frac{1}{p}\|\nabla u\|_{p}^{p}-\frac{b}{q+2}\left\|u^{+}\right\|_{q+2}^{q+2}, \\
& I(u)=\|\nabla u\|_{p}^{p}-b\left\|u^{+}\right\|_{q+2}^{q+2}, \\
& W=\left\{u \in W_{0}^{1, p}(\Omega) \mid I(u)>0, J(u)<d\right\} \cup\{0\},
\end{aligned}
$$

where $u^{+}=\max \{u, 0\}, d=\inf _{u \in W_{0}^{1, p}, u \neq 0}\left(\sup _{\lambda \geq 0} J(\lambda u)\right)$. 
Furthermore, for problem (1.1)-(1.3) and $0<\delta<1$, we define the auxiliary functional $J_{\delta}(u)$ and function $d(\delta)$

$$
\begin{aligned}
& J_{\delta}(u)=\frac{\delta}{p}\|\nabla u\|_{p}^{p}-\frac{b}{q+2}\left\|u^{+}\right\|_{q+2}^{q+2}, \\
& d(\delta)=\frac{1-\delta}{p}\left(\frac{q+2}{p b C_{*}^{q+2}} \delta\right)^{\frac{p}{q-p+2}},
\end{aligned}
$$

where $C_{*}=\sup \frac{\|u\|_{q+2}}{\|\nabla u\|_{p}}$.

In the paper, we always assume that $p$ and $q$ satisfy $(\mathrm{H})$ :

$$
p<2+q<\infty \quad \text { if } N \leq p ; \quad p<2+q<\frac{N p}{N-p} \quad \text { if } N>p .
$$

Before giving our main results, we show some preliminary lemmas which are very important in the following proofs. As for the proofs of these several lemmas, we will not repeat them again (see $[11,20])$.

Lemma 1 ([11], Lemma 2.2) For any given $u \in W_{0}^{1, p}(\Omega),\left\|u^{+}\right\|_{q+2} \neq 0, g(\lambda)=J(\lambda u)$ possesses the following properties:

(1) $\lim _{\lambda \rightarrow 0} g(\lambda)=0, \lim _{\lambda \rightarrow+\infty} g(\lambda)=-\infty$;

(2) There exists a unique $\bar{\lambda}=\bar{\lambda}(u)>0$ such that $g^{\prime}(\bar{\lambda})=0$;

(3) $g^{\prime}(\lambda)>0$, i.e. $g(\lambda)$ is increasing for $0<\lambda<\bar{\lambda} ; g^{\prime}(\lambda)<0$, i.e. $g(\lambda)$ is decreasing for $\bar{\lambda}<\lambda<+\infty$

(4) $g^{\prime \prime}(\lambda)<0$.

Lemma 2 ([20], Lemmas 2.1-2.3) The following sufficient and necessary conditions always hold:

(1) Let $J(u) \leq d(\delta)$, then $J_{\delta}(u)>0$ if and only if $0<\|\nabla u\|_{p}<\left(\frac{q+2}{p b C_{*}^{q+2}} \delta\right)^{\frac{1}{q-p+2}}$.

(2) Let $J(u) \leq d(\delta)$, then $J_{\delta}(u)<0$ if and only if $\|\nabla u\|_{p}>\left(\frac{q+2}{p b C_{*}^{q+2}} \delta\right)^{\frac{1}{q-p+2}}$.

(3) Let $J(u)=d(\delta)$, then $J_{\delta}(u)=0$ if and only if $\|\nabla u\|_{p}=\left(\frac{q+2}{p b C_{*}^{q+2}} \delta\right)^{\frac{1}{q-p+2}}$.

Lemma 3 ([20], Lemma 2.4) The function $d(\delta)$ possesses the following properties on the interval $0 \leq \delta \leq 1$ :

(1) $d(0)=d(1)=0$;

(2) $d(\delta)$ takes the maximum $d\left(\delta_{0}\right)=\frac{1}{\alpha b^{\beta} C_{*}^{\alpha}}$ at $\delta_{0}=\frac{p}{q+2}$, where $\alpha=\frac{p(q+2)}{q-p+2}, \beta=\frac{p}{q-p+2}$;

(3) $d(\delta)$ is increasing on $\left[0, \delta_{0}\right]$ and decreasing on $\left[\delta_{0}, 1\right]$;

(4) For any given $e \in\left(0, d\left(\delta_{0}\right)\right)$, the equation $d(\delta)=e$ has exactly two roots $\delta_{1} \in\left(0, \delta_{0}\right)$ and $\delta_{2} \in\left(\delta_{0}, 1\right)$.

Lemma 4 ([20], Lemma 2.5) $d(\delta)=\inf J(u)$, where $u \in W_{0}^{1, p}(\Omega),\|\nabla u\|_{p} \neq 0, J_{\delta}(u)=0$.

Proposition $1 d=d\left(\delta_{0}\right)=\inf (J(u))$, where $u \in W_{0}^{1, p}(\Omega),\|\nabla u\|_{p} \neq 0, I(u)=0$.

Proof The result can easily be obtained by Lemma 4 and the fact that $J_{\delta_{0}}(u)=0$ is equivalent to $I(u)=0$. 
Now we can define a family of potential wells as follows:

$$
\begin{aligned}
& W_{\delta}=\left\{u \in W_{0}^{1, p}(\Omega) \mid J_{\delta}(u)>0, J(u)<d(\delta)\right\} \cup\{0\}, \quad 0<\delta<1, \\
& \bar{W}_{\delta}=W_{\delta} \cup \partial W_{\delta}=\left\{u \in W_{0}^{1, p}(\Omega) \mid J_{\delta}(u) \geq 0, J(u) \leq d(\delta)\right\} .
\end{aligned}
$$

Obviously, we have $W_{\delta_{0}}=W$.

Remark 1 From $J(u)=\frac{1-\delta}{p}\|\nabla u\|_{p}^{p}+J_{\delta}(u)$, we see that $J_{\delta}(u)>0$ implies that $J(u)>0$.

In the following, we define

$$
\begin{aligned}
& V_{\delta}=\left\{u \in W_{0}^{1, p}(\Omega) \mid J_{\delta}(u)<0, J(u)<d(\delta)\right\}, \quad 0<\delta<1, \\
& \bar{V}_{\delta}=V_{\delta} \cup \partial V_{\delta}=\left\{u \in W_{0}^{1, p}(\Omega) \mid J_{\delta}(u) \leq 0, J(u) \leq d(\delta)\right\}, \\
& V=\left\{u \in W_{0}^{1, p}(\Omega) \mid I(u)<0, J(u)<d\right\}, \\
& B_{\delta}=\left\{u \in W_{0}^{1, p} \mid\|\nabla u\|_{p}<\left(\frac{q+2}{p b C_{*}^{q+2}} \delta\right)^{\frac{1}{q-p+2}}\right\}, \\
& \bar{B}_{\delta}=B_{\delta} \cup \partial B_{\delta}=\left\{u \in W_{0}^{1, p} \mid\|\nabla u\|_{p} \leq\left(\frac{q+2}{p b C_{*}^{q+2}} \delta\right)^{\frac{1}{q-p+2}}\right\}, \\
& B_{\delta}^{c}=\left\{u \in W_{0}^{1, p} \mid\|\nabla u\|_{p}>\left(\frac{q+2}{p b C_{*}^{q+2}} \delta\right)^{\frac{1}{q-p+2}}\right\} .
\end{aligned}
$$

Obviously, we have $V_{\delta_{0}}=V$.

Note that $J(u) \leq \frac{1}{p}\|\nabla u\|_{p}^{p}$, hence for any given $\delta \in(0,1)$, when $0<\|\nabla u\|_{p}<(1-\delta)^{\frac{1}{2}} \times$ $\left(\frac{q+2}{p b C_{*}^{q+2}} \delta\right)^{\frac{1}{q-p+2}}$, we have $J(u)<d(\delta)$ and $J_{\delta}(u)>0$. This implies that $B_{\bar{\delta}} \subset W_{\delta}$, where $\bar{\delta}$ satisfies $\left(\frac{q+2}{p b C_{*}^{q+2}} \bar{\delta}\right)^{\frac{1}{q-p+2}}=(1-\delta)^{\frac{1}{2}}\left(\frac{q+2}{p b C_{*}^{q+2}} \delta\right)^{\frac{1}{q-p+2}}$.

Lemma 5 ([20], Theorem 2.7) Suppose that $W_{\delta}, V_{\delta}, B_{\delta}, B_{\delta}^{c}$, and $\bar{\delta}$ are defined as the above, then

$$
B_{\bar{\delta}} \subset W_{\delta} \subset B_{\delta}, \quad V_{\delta} \subset B_{\delta}^{c} .
$$

Lemma 6 ([20], Lemma 2.10) Assume that $0<J(u)<d$ for some given $u \in W_{0}^{1, p}(\Omega), \delta_{1}<\delta_{2}$ are the two roots of the equation $d(\delta)=J(u)$, then the sign of $J_{\delta}(u)$ is not changed for $\delta \in$ $\left(\delta_{1}, \delta_{2}\right)$.

Lemma 7 ([11], Lemma 2.8) Let $p$ and $q$ satisfy $(\mathrm{H})$, then the solutions given in Theorem 1 satisfy

$$
\int_{0}^{t}\left\|u_{t}\right\|_{m}^{m} d \tau+J(u) \leq J\left(u_{0}\right), \quad \forall t \geq 0
$$

\section{Existence of global weak solutions}

In this section, we obtain the global existence of solutions for problem (1.1)-(1.3) by combining the Galerkin approximation technique and the theory of a family of potential wells. 
Theorem 1 Assume that $p$ and $q$ satisfy $(\mathrm{H}), u_{0}(x) \in W_{0}^{1, p}(\Omega)$. If $0<J\left(u_{0}\right)<d, \delta_{1}<\delta_{2}$ are the two roots of the equation $d(\delta)=J\left(u_{0}\right)$ and $J_{\delta_{2}}\left(u_{0}\right)>0$ or $\left\|\nabla u_{0}\right\|_{p}=0$, then problem (1.1a)-(1.3) admits a global weak solution $u(x, t)$ such that $u \in L^{\infty}\left(0, \infty ; W_{0}^{1, p}(\Omega)\right)$, $u_{t} \in L^{m}\left(0, \infty ; L^{m}(\Omega)\right)$ and $u \in W_{\delta}$ for $\delta \in\left(\delta_{1}, \delta_{2}\right)$ and $0 \leq t<\infty$. Furthermore, we have

(1) $\|u(x, t)\|_{m} \leq\|u(x, s)\|_{m}$ for $t \geq s \geq 0$;

(2) If $N<p$, then the solution is uniquely determined by the initial function;

(3) If $u_{0}(x) \geq 0$ a.e. in $\Omega$, the solution $u(x, t) \geq 0$ a.e. in $\Omega$ for any fixed $t>0$, hence $u(x, t)$ is a solution of the problem (1.1)-(1.3).

Proof Let $\left\{\omega_{j}(x)\right\}$ be a system of base functions of $W_{0}^{1, p}(\Omega)$. Construct approximate solutions $u_{n}(x, t)$ in the form

$$
u_{n}(t)=\sum_{j=1}^{n} g_{j n}(t) \omega_{j}(x), \quad n=1,2, \ldots,
$$

satisfying

$$
\begin{aligned}
& \left(\left|u_{n t}\right|^{m-2} u_{n t}, \omega_{s}\right)+\left(\left|\nabla u_{n}\right|^{p-2} \nabla u_{n}, \nabla \omega_{s}\right)=\left(b u_{n}^{q+1}, \omega_{s}\right), \quad s=1,2, \ldots, n, \\
& u_{n}(x, 0)=\sum_{j=1}^{n} a_{j n} \omega_{j}(x) \rightarrow u_{0}(x), \quad \text { in } W_{0}^{1, p}(\Omega) .
\end{aligned}
$$

Multiplying (1.1a) by $g_{s n}^{\prime}(t)$, summing over $s$ and integrating with respect to $t$, we obtain

$$
\begin{aligned}
J_{n}(t) & =\frac{1}{p}\left\|\nabla u_{n}\right\|_{p}^{p}-\frac{b}{q+2}\left\|u_{n}^{+}\right\|_{q+2}^{q+2}+\int_{0}^{t}\left\|u_{n t}\right\|_{m}^{m} d \tau \\
& =\frac{1}{p}\left\|\nabla u_{n}(0)\right\|_{p}^{p}-\frac{b}{q+2}\left\|u_{n}^{+}(0)\right\|_{q+2}^{q+2}=J_{n}\left(u_{0}\right) .
\end{aligned}
$$

Note that $J_{\delta_{2}}\left(u_{0}\right)>0$ implies $\left\|\nabla u_{0}\right\|_{p} \neq 0$. By Lemma 6, we have $J_{\delta}\left(u_{0}\right)>0$ for $\delta \in\left(\delta_{1}, \delta_{2}\right)$. From this and $J\left(u_{0}\right)=d\left(\delta_{1}\right)=d\left(\delta_{2}\right)<d(\delta)$, we obtain $u_{0}(x) \in W_{\delta}$ for $\delta \in\left(\delta_{1}, \delta_{2}\right)$. If $\left\|\nabla u_{0}\right\|_{p}=$ 0 , then $u_{0}(x) \in W_{\delta}$ for $\delta \in(0,1)$. For any fixed $\delta \in\left(\delta_{1}, \delta_{2}\right)$, we have $J_{\delta}\left(u_{n}(0)\right)>0$ and $J_{n}\left(u_{0}\right)<$ $d(\delta)$ (if $J_{\delta_{2}}\left(u_{0}\right)>0$ ) or $u_{n}(0) \in B_{\bar{\delta}}$ (if $\left\|\nabla u_{0}\right\|_{p}=0$ and $\bar{\delta}$ is defined in Lemma 5), thereby $u_{n}(0) \in W_{\delta}$ for sufficiently large $n$.

Next, we prove that $u_{n}(t) \in W_{\delta}$ for sufficiently large $n$ and $t>0$. Otherwise, there must be a $t_{0}>0$ such that $u_{n}\left(t_{0}\right) \in \partial W_{\delta}$, i.e. $J_{\delta}\left(u_{n}\left(t_{0}\right)\right)=0$ and $\left\|\nabla u_{n}\left(t_{0}\right)\right\|_{p} \neq 0$ or $J\left(u_{n}\left(t_{0}\right)\right)=d(\delta)$. From (3.1), we have

$$
J\left(u_{n}(t)\right) \leq J_{n}\left(u_{0}\right)<d(\delta), \quad t>0,
$$

hence $J\left(u_{n}\left(t_{0}\right)\right)=d(\delta)$ is impossible. If $J_{\delta}\left(u_{n}\left(t_{0}\right)\right)=0$ and $\left\|\nabla u_{n}\left(t_{0}\right)\right\|_{p} \neq 0$, then by Lemma 4 , we have $J\left(u_{n}\left(t_{0}\right)\right) \geq d(\delta)$, which is also impossible. Thus from (3.1) and Lemma 2, we obtain

$$
\begin{aligned}
& \left\|\nabla u_{n}(t)\right\|_{p}<\left(\frac{q+2}{p b C_{*}^{q+2}} \delta\right)^{\frac{1}{q-p+2}} \\
& \left\|u_{n}(t)\right\|_{q+2}<C_{*}\left\|\nabla u_{n}(t)\right\|_{p}<C_{*}\left(\frac{q+2}{p b C_{*}^{q+2}} \delta\right)^{\frac{1}{q-p+2}}
\end{aligned}
$$


and

$$
\int_{0}^{t}\left\|u_{n t}(\tau)\right\|_{m}^{m} d \tau<d(\delta),
$$

for $t>0$ and sufficiently large $n$. From these and the compactness method, we can prove that problem (1.1a)-(1.3) admits a global weak solution $u(x, t)$ such that $u \in$ $L^{\infty}\left(0, \infty ; W_{0}^{1, p}(\Omega)\right), u_{t} \in L^{m}\left(0, \infty ; L^{m}(\Omega)\right)$ and $u \in W_{\delta}$ for any $\delta \in\left(\delta_{1}, \delta_{2}\right)$ and $0 \leq t<\infty$.

Furthermore, by Theorem 1 in [10] we can easily get the results (1)-(3), here we omit the proofs.

Similarly, we can get the following conclusions directly.

Corollary 1 Under the conditions of Theorem 1 , we have $u \in \bar{W}_{\delta_{1}}$ for $0 \leq t<\infty$.

Corollary 2 If the assumption $I\left(u_{0}\right)>0$ or $\left\|\nabla u_{0}\right\|_{p}=0$ is replaced by $J_{\delta_{2}}\left(u_{0}\right)>0$ or $\left\|\nabla u_{0}\right\|_{p}=0$, i.e. $u_{0}(x) \in W$, then the conclusion of Theorem 1 also holds.

Corollary 3 If the assumption $u_{0}(x) \in B_{\delta_{2}}$ is replaced by $J_{\delta_{2}}\left(u_{0}\right)>0$ or $\left\|\nabla u_{0}\right\|_{p}=0$, then problem (1.1)-(1.3) admits a global weak solution $u(x, t)$ such that $u \in L^{\infty}\left(0, \infty ; W_{0}^{1, p}(\Omega)\right)$, $u_{t} \in L^{m}\left(0, \infty ; L^{m}(\Omega)\right)$ and $u \in \bar{B}_{\delta_{1}}$ for $0 \leq t<\infty$.

\section{Invariant property and vacuum isolating of global solutions}

In this section, we discuss the invariance of some sets under the flow of (1.1)-(1.3) and vacuum isolating behavior of solutions for problem (1.1)-(1.3).

\subsection{Invariant property of global solutions}

Theorem 2 Assume that $p$ and $q$ satisfy $(\mathrm{H}), 0 \leq u_{0}(x) \in W_{0}^{1, p}(\Omega)$. If $0<e<d, \delta_{1}<\delta_{2}$ are the two roots of the equation $d(\delta)=e$, then the following hold.

(1) All solutions of problem (1.1)-(1.3) with initial energy $0<J\left(u_{0}\right) \leq$ e belong to $\bar{W}_{\delta}$ for $\delta \in\left(\delta_{1}, \delta_{2}\right)$, provided that $I\left(u_{0}\right)>0$ or $\left\|\nabla u_{0}\right\|_{p}=0$.

(2) All solutions of problem (1.1)-(1.3) with initial energy $0<J\left(u_{0}\right) \leq$ e belong to $\bar{V}_{\delta}$ for $\delta \in\left(\delta_{1}, \delta_{2}\right)$, provided that $I\left(u_{0}\right)<0$.

Proof Firstly, we consider the case of $J\left(u_{0}\right)=e$. Here we denote $u(x, t) \triangleq u(t)$.

(1) Let $u(t)$ be any solution of problem (1.1)-(1.3) with initial energy $J\left(u_{0}\right)=e$ and $I\left(u_{0}\right)>0$ or $\left\|\nabla u_{0}\right\|_{p}=0, T$ be the existence time of $u(t)$. Since

$$
\begin{aligned}
J_{\delta_{0}}\left(u_{0}\right) & =\frac{\delta_{0}}{p}\left\|\nabla u_{0}\right\|_{p}^{p}-\frac{b}{q+2}\left\|u_{0}\right\|_{q+2}^{q+2} \\
& =\frac{1}{q+2}\left\|\nabla u_{0}\right\|_{p}^{p}-\frac{b}{q+2}\left\|u_{0}\right\|_{q+2}^{q+2}=\frac{1}{q+2} I\left(u_{0}\right)>0,
\end{aligned}
$$

by Theorem 1 , we have $u_{0}(x) \in W_{\delta}$.

Next we prove $u(t) \in W_{\delta}$ for $\delta \in\left(\delta_{1}, \delta_{2}\right), 0<t<T$. Otherwise, there exists a $t_{0} \in(0, T)$ such that $u\left(t_{0}\right) \in \partial W_{\delta}$ for some $\delta \in\left(\delta_{1}, \delta_{2}\right)$, i.e. $J_{\delta}\left(u\left(t_{0}\right)\right)=0,\left\|\nabla u\left(t_{0}\right)\right\|_{p} \neq 0$ or $J\left(u\left(t_{0}\right)\right)=d(\delta)$. From Lemma 7

$$
J(u)+\int_{0}^{t}\left\|u_{t}\right\|_{m}^{m} d \tau \leq J\left(u_{0}\right)<d(\delta),
$$


we see that $J\left(u\left(t_{0}\right)\right)=d(\delta)$ is impossible. On the other hand, if $J_{\delta}\left(u\left(t_{0}\right)\right)=0$ and $\left\|\nabla u\left(t_{0}\right)\right\|_{p} \neq$ 0 , then by Lemma 4 , we have $J\left(u\left(t_{0}\right)\right) \geq d(\delta)$, which contradicts (4.1).

(2) Let $u(t)$ be any solution of problem (1.1)-(1.3) with initial energy $J\left(u_{0}\right)=e$ and $I\left(u_{0}\right)<$ $0, T$ be the existence time of $u(t)$. Since the sign of $J_{\delta}\left(u_{0}\right)$ is not changed for $\delta \in\left(\delta_{1}, \delta_{2}\right)$, we have $J_{\delta}\left(u_{0}\right)<0$ for $\delta \in\left(\delta_{1}, \delta_{2}\right)$. From this and $J\left(u_{0}\right)<d(\delta)$ for $\delta \in\left(\delta_{1}, \delta_{2}\right)$ we obtain $u_{0}(x) \in V_{\delta}$ for $\delta \in\left(\delta_{1}, \delta_{2}\right)$.

Next we prove $u(t) \in V_{\delta}$ for $\delta \in\left(\delta_{1}, \delta_{2}\right)$ and $0<t<T$. Otherwise, there exists a $t_{0} \in(0, T)$ such that $u\left(t_{0}\right) \in \partial V_{\delta}$ for some $\delta \in\left(\delta_{1}, \delta_{2}\right)$, i.e. $J_{\delta}\left(u\left(t_{0}\right)\right)=0$ or $J\left(u\left(t_{0}\right)\right)=d(\delta)$. From (4.1) we see that $J\left(u\left(t_{0}\right)\right)=d(\delta)$ is impossible. On the other hand, let $t_{0}$ be the first time such that $J_{\delta}\left(u\left(t_{0}\right)\right)=0$, then $J_{\delta}(u(t))<0$ for $0 \leq t<t_{0}$. From (4.1) and Lemma 2, we have $\|\nabla u(t)\|_{p}>$ $\left(\frac{q+2}{p b C_{*}^{q+2}} \delta\right)^{\frac{1}{q-p+2}}$ for $0 \leq t<t_{0}$. Hence we have $\left\|\nabla u\left(t_{0}\right)\right\|_{p}>\left(\frac{q+2}{p b C_{*}^{q+2}} \delta\right)^{\frac{1}{q-p+2}}$, thus by Lemma 4, we get $J\left(u\left(t_{0}\right)\right) \geq d(\delta)$, which contradicts (4.1).

For the case of $0<J\left(u_{0}\right)<e$, we can obtain the same results as the case $J\left(u_{0}\right)=e$ by Lemma 4 , we omit it here.

Remark 2 Assume that $0<J\left(u_{0}\right) \leq e$, then $W_{\delta}$ and $V_{\delta}$ are invariant under the flow of (1.1)-(1.3) for any $\delta \in\left(\delta_{1}, \delta_{2}\right)$.

From the above Theorem 2 and Lemma 2, we can easily get the following conclusions.

Theorem 3 Let $p$ and q satisfy $(\mathrm{H}), 0 \leq u_{0}(x) \in W_{0}^{1, p}(\Omega)$. Assume that $0<e<d, \delta_{1}<\delta_{2}$ are the two roots of the equation $d(\delta)=e$, then the following hold.

(1) All solutions of problem (1.1)-(1.3) with initial energy $0<J\left(u_{0}\right) \leq e$ and $u_{0}(x) \in B_{\delta_{0}}$ belong to $\bar{B}_{\delta_{1}}$ for $\delta \in\left(\delta_{1}, \delta_{2}\right)$.

(2) All solutions of problem (1.1)-(1.3) with initial energy $0<J\left(u_{0}\right) \leq e$ and $u_{0}(x) \in B_{\delta_{0}}^{c}$ belong to $\bar{B}_{\delta_{2}}^{c}$ for $\delta \in\left(\delta_{1}, \delta_{2}\right)$.

Remark 3 Let $p$ and $q$ satisfy $(\mathrm{H}), 0 \leq u_{0}(x) \in W_{0}^{1, p}(\Omega)$. Assume that $0<J\left(u_{0}\right) \leq e$, then $B_{\delta}$ and $B_{\delta}^{c}$ are invariant under the flow of (1.1)-(1.3) for any $\delta \in\left(\delta_{1}, \delta_{2}\right)$.

\subsection{Vacuum isolating of global solutions}

The result of Theorem 3 shows that for any given $e \in(0, d)$, there exists a corresponding vacuum region of solutions

$$
U_{e}=\left\{u \in W_{0}^{1, p}(\Omega) \mid\left(\frac{q+2}{p b C_{*}^{q+2}} \delta_{1}\right)^{\frac{1}{q-p+2}}<\|\nabla u\|_{p}<\left(\frac{q+2}{p b C_{*}^{q+2}} \delta_{2}\right)^{\frac{1}{q-p+2}}\right\}
$$

for the set of all solutions of problem (1.1)-(1.3) with initial energy $J\left(u_{0}\right)$ satisfying $0<$ $J\left(u_{0}\right) \leq e$, there is no solution in $U_{e}$ and all solutions are isolated by $U_{e}$. This phenomenon is called the phenomenon of vacuum isolating of solutions. Obviously, the vacuum region $U_{e}$ of solutions becomes bigger and bigger with decreasing of $e$. As the limit case $e=0$, we obtain the biggest vacuum region of solutions (for $J\left(u_{0}\right) \geq 0$ )

$$
U_{0}=\left\{u \in W_{0}^{1, p}(\Omega) \mid 0<\|\nabla u\|_{p}<\left(\frac{q+2}{p b C_{*}^{q+2}}\right)^{\frac{1}{q-p+2}}\right\} .
$$


Theorem 4 Let $p$ and $q$ satisfy $(\mathrm{H}), 0 \leq u_{0}(x) \in W_{0}^{1, p}(\Omega)$. All nontrivial solutions of problem (1.1)-(1.3) with initial energy $J\left(u_{0}\right)=0$ lie outside of the ball $B_{1}$ (maybe in $\left.\partial B_{1}\right)$.

Theorem 5 Let $p$ and $q$ satisfy $(\mathrm{H}), 0 \leq u_{0}(x) \in W_{0}^{1, p}(\Omega)$. All nontrivial solutions of problem (1.1)-(1.3) with initial energy $J\left(u_{0}\right)<0$ satisfy

$$
\|\nabla u\|_{p}>\left(\frac{q+2}{p b C_{*}^{q+2}}\right)^{\frac{1}{q-p+2}}
$$

and

$$
\|\nabla u\|_{p} \geq\left[\frac{(q+2) \sqrt{\frac{-4 J\left(u_{0}\right)}{p}}}{b C_{*}^{q+2}}\right]^{\frac{2}{2 q-p+4}} .
$$

Remark 4 The proofs of Theorems 4-5 are similar to Theorems 4.7-4.8 in [20], we omit them.

\section{Nonexistence of global solutions}

In this section, we given the sufficient condition of global nonexistence of solutions.

Theorem 6 Assume that $2<m<2+q, u_{0}(x) \in W_{0}^{1, p}(\Omega), u(x, t)$ is a local solution of problem (1.1)-(1.3) on $[0, T]$, then no solution of $(1.1)-(1.3)$ can exist on $[0, \infty)$ when $J\left(u_{0}\right)<0$.

Proof Assume for contradiction that there is a solution of (1.1)-(1.3) on $[0, \infty)$.

Define

$$
H(t)=\int_{0}^{t}\left\|u_{t}\right\|_{m}^{m} d \tau-J\left(u_{0}\right)
$$

hence

$$
\|u\|_{q+2}^{q+2}=\frac{q+2}{b}\left[\frac{1}{p}\|\nabla u\|_{p}^{p}+H(t)\right] \geq \frac{q+2}{b} H(t) \geq-\frac{q+2}{b} J\left(u_{0}\right)>0 .
$$

Multiplying (1.1) by $u$ and integrating over $\Omega$, we have

$$
\begin{aligned}
0 & =b\|u\|_{q+2}^{q+2}-\|\nabla u\|_{p}^{p}-\left(\left|u_{t}\right|^{m-2} u_{t}, u\right) \\
& =\frac{b(q-p+2)}{q+2}\|u\|_{q+2}^{q+2}-p J(u(t))-\left(\left|u_{t}\right|^{m-2} u_{t}, u\right) \\
& \geq \frac{b(q-p+2)}{q+2}\|u\|_{q+2}^{q+2}-p J(u(t))-\left\|u_{t}\right\|_{\frac{(m-1)(q+2)}{q+1}}^{m-1}\|u\|_{q+1} .
\end{aligned}
$$

As $J(u(t))<J\left(u_{0}\right)<0$, we see from (5.1) and (5.2) that

$$
\left\|u_{t}\right\|_{\frac{(m-1)(q+2)}{q+1}}^{m-1} \geq \frac{b(q-p+2)}{q+2}\|u\|_{q+2}^{q+1} \geq \frac{b(q-p+2)}{q+2}\left[\frac{q+2}{b} H(t)\right]^{\frac{q+1}{q+2}} .
$$

Since $m<2+q$, we have $m \geq \frac{(m-1)(q+2)}{q}$. By the embedding theorem and (5.3), we have

$$
H^{\prime}(t)=\left\|u_{t}\right\|_{m}^{m} \geq C\left\|u_{t}\right\|_{\frac{(m-1)(q+2)}{q+1}}^{q+1} \geq C(H(t))^{\frac{(q+1) m}{(m-1)(q+2)}} .
$$


Since $m<2+q$, we have

$$
\frac{(q+1) m}{(m-1)(q+2)}-1=\frac{q-m+2}{(m-1)(q+2)}>0 .
$$

Hence we write $\frac{q m}{(m-1)(q+2)}=1+\theta, \theta>0$, then

$$
\frac{H^{\prime}(t)}{H^{1+\theta}}>C
$$

By setting $H_{0}=H(0)=-J\left(u_{0}\right)>0$, we obtain

$$
\frac{1}{\theta H_{0}^{\theta}} \geq \frac{1}{\theta(H(t))^{\theta}}+C t
$$

This is impossible, since the left hand side is finite and the right hand side goes to $\infty$ as $t \rightarrow \infty$.

\section{Existence of global solution with critical initial conditions}

In this section, we prove the result of global existence with critical initial conditions.

Theorem 7 Assume that $p$ and $q$ satisfy $(\mathrm{H}), 0 \leq u_{0}(x) \in W_{0}^{1, p}(\Omega)$. If $J\left(u_{0}\right)=d(\delta), J_{\delta_{1}}\left(u_{0}\right)>$ 0 or $J_{\delta_{1}}\left(u_{0}\right)=0,0<J\left(u_{0}\right) \leq d(\delta), \delta_{1}<\delta_{2}$ are the two roots of the equation $J\left(u_{0}\right)=d(\delta)$, then problem (1.1)-(1.3) admits a global solution $u(x, t)$ such that $u \in L^{\infty}\left(0, \infty ; W_{0}^{1, p}(\Omega)\right)$, $u_{t} \in L^{m}\left(0, \infty ; L^{m}(\Omega)\right)$ and $u \in \bar{W}_{\delta}$ for any $\delta \in\left(\delta_{1}, \delta_{2}\right)$ and $0 \leq t<\infty$, where $\bar{W}_{\delta}=W_{\delta} \cup$ $\partial W_{\delta}=\left\{u \in W_{0}^{1, p}(\Omega) \mid J_{\delta}(u) \geq 0, J(u) \leq d(\delta)\right\}$.

Proof Let $\lambda_{n}=1-\frac{1}{n}, u_{0 n}(x)=\lambda_{n} u_{0}(x), n=2,3, \ldots$ Consider the initial condition

$$
u(x, 0)=u_{0 n}(x)
$$

with the corresponding problem (1.1)-(1.3) and suppose that $\delta_{1}^{n}<\delta_{2}^{n}$ are two roots of the equation $J\left(u_{0 n}\right)=d(\delta)$.

Since $J_{\delta_{1}}\left(u_{0}\right) \geq 0$, we obtain $\frac{\delta_{1}}{p}\left\|\nabla u_{0}\right\|_{p}^{p}-\frac{b}{q+2}\left\|u_{0}\right\|_{q+2}^{q+2} \geq 0$, i.e. $\left[\frac{\delta_{1}(q+2)\left\|\nabla u_{0}\right\|_{p}^{p}}{b p\left\|u_{0}\right\|_{q+2}^{q+2}}\right]^{\frac{1}{q-p+2}} \geq 1$. Thus $\bar{\lambda}=\left(\frac{\left\|\nabla u_{0}\right\|_{p}^{p}}{b p\left\|u_{0}\right\|_{q+2}^{q+2}}\right)^{\frac{1}{q-p+2}} \geq\left(\frac{1}{\delta_{1}} \cdot \frac{p}{q+2}\right)^{\frac{1}{q-p+2}} \geq 1$. From $0<\lambda_{n}<1 \leq \bar{\lambda}$ and Lemma 1 , we get

$$
\begin{aligned}
& J\left(u_{0 n}\right)=J\left(\lambda_{n} u_{0}\right)<J\left(u_{0}\right) \leq d(\delta) \leq d, \\
& J_{\delta_{1}}\left(u_{0 n}\right)=J_{\delta_{1}}\left(\lambda_{n} u_{0}\right)>0 .
\end{aligned}
$$

Obviously, $J(u)=\frac{1-\delta}{p}\|\nabla u\|_{p}^{p}+J_{\delta}(u)$ implies that $J\left(u_{0 n}\right)>0$. As $\delta_{2}^{n}>\delta_{0}>\delta_{1}$ and $J_{\delta}(u)$ is increasing with $\delta$, it follows that $J_{\delta_{2}^{n}}\left(u_{0 n}\right)>0$.

Thus, by Theorem 1 , the problem admits a global solution $u_{n}(x, t)$ such that $u_{n} \in$ $L^{\infty}\left(0, \infty ; W_{0}^{1, p}(\Omega)\right), u_{n t} \in L^{m}\left(0, \infty ; L^{m}(\Omega)\right)$ and $u_{n} \in W_{\delta}$ for any $\delta \in\left(\delta_{1}^{n}, \delta_{2}^{n}\right)$, satisfying

$$
\begin{aligned}
& \left(\left|u_{n t}\right|^{m-2} u_{n t}, v\right)+\left(\left|\nabla u_{n}\right|^{p-2} \nabla u_{n}, \nabla v\right)=\left(b u_{n}^{q+1}, v\right), \quad \forall v \in W_{0}^{1, p}(\Omega), t \geq 0, \\
& \int_{0}^{t}\left\|u_{n t}\right\|_{m}^{m} d \tau+J\left(u_{n}\right) \leq J\left(u_{n}(0)\right)=J\left(u_{0 n}\right)<d(\delta), \quad t \geq 0,
\end{aligned}
$$


i.e.

$$
\int_{0}^{t}\left\|u_{n t}\right\|_{m}^{m} d \tau+\frac{1-\delta}{p}\left\|\nabla u_{n}\right\|_{p}^{p}+J_{\delta}\left(u_{n}\right)<d(\delta), \quad t \geq 0 .
$$

From $J_{\delta}\left(u_{n}\right) \geq 0$, we obtain

$$
\int_{0}^{t}\left\|u_{n t}\right\|_{m}^{m} d \tau<d, \quad \frac{1-\delta}{p}\left\|\nabla u_{n}\right\|_{p}^{p}<d, \quad t \geq 0
$$

Hence there exist $u, \xi$, and subsequence $\left\{u_{v}\right\}$ of $\left\{u_{n}\right\}$ such that

$$
\begin{aligned}
& u_{v} \rightarrow u \quad \text { in } L^{\infty}\left(0, \infty ; W_{0}^{1, p}(\Omega)\right) \text { weak star, } \\
& u_{v t} \rightarrow u \quad \text { in } L^{m}\left(0, \infty ; L^{m}(\Omega)\right) \text { weakly, } \\
& b u_{v}^{q+1} \rightarrow \xi \quad \text { in } L^{\infty}\left(0, \infty ; L^{(q+2)^{\prime}}(\Omega)\right) \text { weak star. }
\end{aligned}
$$

By using the monotone operator method, we get $\xi=b u^{q+1}$.

In (6.1), letting $n=v \rightarrow \infty$ we obtain

$$
\left(\left|u_{t}\right|^{m-2} u_{t}, v\right)+\left(|\nabla u|^{p-2} \nabla u, \nabla v\right)=\left(b u^{q+1}, v\right), \quad \forall v \in W_{0}^{1, p}(\Omega), t \geq 0 .
$$

On the other hand, letting $n=v \rightarrow \infty$ in $u_{n}(x, 0)=u_{0 n}(x)$ we get $u(x, 0)=u_{0}(x)$ in $W_{0}^{1, p}(\Omega)$. Also $\delta_{1}^{n} \rightarrow \delta_{1}, \delta_{2}^{n} \rightarrow \delta_{2}$ as $n \rightarrow \infty$.

Furthermore, from (6.2) and Lemma 7, we have $J(u) \leq d(\delta)$ for $0 \leq t<\infty$. On the other hand, from (6.3) we have

$$
\|\nabla u\|_{p}^{p} \leq \lim _{\nu \rightarrow \infty}\left\|\nabla u_{\nu}\right\|_{p}^{p} \leq \frac{p}{1-\delta} \cdot d(\delta)=\left(\frac{q+2}{p b C_{*}^{q+2}} \delta\right)^{\frac{p}{q-p+2}} .
$$

By Lemma 2 , we have $J_{\delta}(u) \geq 0$ and $u \in \bar{W}_{\delta}$ for any $\delta \in\left(\delta_{1}, \delta_{2}\right)$.

Remark 5 The invariant sets and vacuum of solutions for problem (1.1)-(1.3) with critical initial conditions also occur.

Remark 6 Taking $m=2$ or $p=2$, Theorem 7 is still satisfied and generalizes the results of [11]. Similarly, the invariant sets and vacuum isolating of solutions also occur.

Remark 7 In fact, all the results in our paper also hold for the homogeneous Dirichlet initial boundary value problem for the more general equation

$$
\left|u_{t}\right|^{m-2} u_{t}=\operatorname{div}\left(|\nabla u|^{p-2} \nabla u\right)+b|u|^{q} u
$$




\section{Author details}

'Department of Mathematics, Pusan National University, Busan, 609-735, Republic of Korea. ${ }^{2}$ School of Mathematical Sciences, Ocean University of China, Qingdao, 266100, P.R. China.

\section{Acknowledgements}

The second and third authors were supported by Basic Science Research Program through the National Research Foundation of Korea (NRF) funded by the Ministry of Education, Science and Technology (No. 2012005767) and the National Science Foundation of Shandong Province of China (ZR2012AM018) and the Fundamental Research Funds for the Central Universities (No. 201362032), respectively. The authors would like to express their sincere gratitude to the anonymous reviewers for their insightful and constructive comments.

\section{Received: 30 October 2013 Accepted: 22 January 2014 Published: 07 Feb 2014}

\section{References}

1. Bebernes, J, Eberly, D: Mathematical problems from combustion theory. Applied Mathematical Sciences, vol. 83. Springer, New York (1989)

2. Pao, CV: Nonlinear parabolic and elliptic equations. Plenum Press, New York (1992)

3. Bourgault, Y, Coudiere, Y, Pierre, C: Existence and uniqueness of the solution for the bidomain model used in cardiac electrophysiology. Nonlinear Anal., Real World Appl. 10, 458-482 (2009)

4. Klainerman, S, Ponce, G: Global, small amplitude solutions to nonlinear evolution equations. Commun. Pure Appl. Math. 36, 133-141 (1983)

5. Gazzola, F, Weth, T: Finite time blow-up and global solutions for semilinear parabolic equations with initial data at high energy level. Differ. Integral Equ. 18, 961-990 (2005)

6. Levine, HA, Park, SR, Serrin, J: Global existence and nonexistence theorems for quasilinear evolution equations of formally parabolic type. J. Differ. Equ. 142, 212-229 (1998)

7. Sattinger, DH: On global solution of nonlinear hyperbolic equations. Arch. Ration. Mech. Anal. 30, 148-172 (1968)

8. Levine, HA, Smith, RA: Ames, A potential well theory for the heat equation with a nonlinear boundary condition. Math. Methods Appl. Sci. 9, 127-136 (1987)

9. Payne, LE, Sattinger, DH: Saddle points and instability of nonlinear hyperbolic equations. Isr. J. Math. 22, 273-303 (1975)

10. Tsutsumi, M: Existence and nonexistence of global solutions for nonlinear parabolic equations. Publ. Res. Inst. Math. Sci. 8, 211-229 (1972/73)

11. Liu, YC, Zhao, JS: Nonlinear parabolic equations with critical initial conditions $J\left(u_{0}\right)=d$ or $/\left(u_{0}\right)=0$. Nonlinear Anal. 58 873-883 (2004)

12. Pang, JS, Zhang, HW: Existence and nonexistence of the global solution on the quasilinear parabolic equation. Chin. Q. J. Math. 22, 444-450 (2007)

13. Todorova, G: Existence of a solution of the wave equation with nonlinear damping and source terms. J. Differ. Equ. 109, 295-308 (1994)

14. Ikehata, R: Some remarks on the wave equations with nonlinear damping and source terms. Nonlinear Anal. TMA 27, 1165-1175 (1996)

15. Ikehata, R, Suzuki, T: Stable and unstable sets for evolution equations of parabolic and hyperbolic type. Hiroshima Math. J. 26, 475-491 (1996)

16. Pucci, P, Serrin, J: Asymptotic stability for nonautonomous dissipative wave systems. Commun. Pure Appl. Math. 49 177-216 (1996)

17. Aassila, M: Global existence and global nonexistence of solutions to a wave equation with nonlinear damping and source terms. Asymptot. Anal. 30, 301-311 (2002)

18. Alfredo, J, Avila, E: A characterization of global and nonglobal solutions of nonlinear wave and Kirchhoff equations. Nonlinear Anal. 52, 1111-1127 (2003)

19. $\mathrm{Xu}, \mathrm{RZ}, \mathrm{CaO}, \mathrm{XY}, \mathrm{Yu}, \mathrm{T}$ : Finite time blow-up and global solutions for a class of semilinear parabolic equations at high energy level. Nonlinear Anal., Real World Appl. 13, 197-202 (2012)

20. Liu, YC: On ponential wells and vacuum isolating of solutions for semilinear wave equations. J. Differ. Equ. 192 , 155-169 (2003)

21. Liu, YC, Zhao, JS: On potential wells and applications to semilinear hyperbolic equations and parabolic equations. Nonlinear Anal. 64, 2665-2687 (2006)

22. Liu, YC, Xu, RZ, Yu, T: Wave equations and reaction-diffusion equations with several nonlinear source terms. Appl. Math. Mech. 28, 1209-1218 (2007)

23. Liu, YC, Xu, RZ: Potential well method for initial boundary value problem of the generalized double dispersion equations. Commun. Pure Appl. Anal. 7, 63-81 (2008)

24. Jiang, XL, Xu, RZ: Global well-posedness for semilinear hyperbolic equations with dissipative term. J. Appl. Math Comput. 38, 467-487 (2012)

25. Wu, ST: Global existence, blow-up and asymptotic behavior of solutions for a class of coupled nonlinear Klein-Gordon equations with damping terms. Acta Appl. Math. 119, 75-95 (2012)

10.1186/1687-2770-2014-33

Cite this article as: Xu et al.: Global existence and nonexistence of solutions for quasilinear parabolic equation. Boundary Value Problems 2014, 2014:33 